\title{
Recurrent Cough Syncope Due to Pertussis in an Adult: A Case Report and Review of the Literature
}

\author{
Hanna Dawood ${ }^{\mathrm{a}}$, Nizar Elias ${ }^{\mathrm{a}}$, Gleb Slobodin ${ }^{\mathrm{a}}$, Majed Odeh ${ }^{\mathrm{a}, \mathrm{b}}$
}

\begin{abstract}
Cough syncope is a well-recognized syndrome in which loss of consciousness usually occurs immediately after prolonged bouts of violent coughing lasting for seconds, with rapid and complete recovery of consciousness. Its pathophysiology is still incompletely understood. Although pertussis is a common cause of cough and cough syncope is not rare, to the best of our knowledge, only five cases of cough syncope related to pertussis have been previously reported, two of which are not well documented. We describe a 52-year-old male who presented to the emergency department with cough and recurrent episodes of cough syncope of 3 months duration due to pertussis which was completely resolved by treatment with azithromycin. Since pertussis is a common cause of subacute and chronic cough in adolescents and adults, and the clinical characteristics of pertussis among adults are less obvious than among children, it seems likely that cough syncope due to pertussis is underdiagnosed. It is important for the physicians to be aware of pertussis as a cause of chronic cough and cough syncope in adults in order to terminate the attacks by adequate antibiotic treatment.
\end{abstract}

Keywords: Cough; Syncope; Pertussis; Adults

\section{Introduction}

The syndrome of cough syncope, also called "tussive syncope", was first described by Charcot in 1876 under the name "laryngeal vertigo". Originally, cough syncope was thought to be a form of epilepsy, and only in 1940s, it was recognized to be of syncopal nature [1]. It is characterized by paroxysmal cough, facial congestion, turgidity and cyanosis. Unconsciousness is followed within seconds to minutes, lasting only for

Manuscript accepted for publication December 02, 2014

aDepartment of Internal Medicine A, Bnai Zion Medical Center, Faculty of Medicine, Technion, Haifa, Israel

${ }^{b}$ Corresponding Author: Majed Odeh, Department of Internal Medicine A, Bnai Zion Medical Center, Golomb 47, Haifa 31048, Israel.

Email: majed.odeh@b-zion.org.il

doi: http://dx.doi.org/10.14740/jmc2010w seconds [2]. It usually occurs while sitting or standing, but may also occur during supine position, and consciousness rapidly recovers completely. It usually occurs in moderately obese, middle-aged men with broncho-pulmonary disorders $[1,2]$. A few case studies have also shown cough syncope induced by gastroesophageal reflux disease [3], intra-cranial tumors [4] and pericarditis [5]. The pathophysiology of cough syncope is still incompletely understood.

Although pertussis is a common cause of cough, to the best of our knowledge, only five cases of cough syncope related to pertussis have been previously reported in the medical literature [6-9]. Two of them are not well documented [9]. We describe a 52-year-old man with recurrent episodes of syncope accompanying cough attacks of 3 months duration due to pertussis.

\section{Case Report}

A 52-year-old man was referred to the emergency department because of paroxysmal productive cough for the last 3 months. During episodes of cough, which occurred several times 1 day, the patient suffered from loss of consciousness for a few seconds. One day before admission, he had an episode of cough followed by syncope with a fall causing head trauma. The patient denied hemoptysis, fever or chills, chest pain or dyspnea, rhinorrhea, sore throat or paroxysmal nocturnal dyspnea. He was treated with amoxicillin/calvulanic acid for 7 days before his referral, but with no clinical improvement. The patient had a history of follicular lymphoma diagnosed 6 years ago. He was treated with cyclophosphamide, rituximab and fludarabine, and was in remission for the last 5 years. One year before his admission, the patient underwent a cardiac evaluation, which included cardiac echography and coronary angiography, which revealed normal findings.

Physical examination revealed normal heart rate, blood pressure, body temperature and oxygen saturation of $97 \%$ on room air. A right peri-orbital laceration with hematoma caused by the fall was observed. Lungs and heart examination was normal, and no enlarged lymph nodes were palpated. Other physical findings were unremarkable.

Routine hematological and biochemical blood tests revealed normal results. Blood, throat and sputum cultures for common pathogens were negative. Chest X-ray film, electro- 
cardiogram (ECG), and head, neck and chest computerized tomography (CT) scan were normal. Laryngeal examination, including optic fiber laryngoscopy was also normal. A continuous $24 \mathrm{~h}$ ECG recording, as well as echocardiography, revealed normal findings. DNA detection by PCR from nasopharyngeal secretions was positive for Bordetella pertussis.

The patient was treated orally with azithromycin for 5 days, bringing to a significant gradual clinical improvement. The frequency and intensity of cough episodes declined, and completely resolved 3 weeks later. No cough orsyncopal attacks occurred during a follow-up period of 3 months.

\section{Discussion}

Syncope is a transient, self-limited loss of consciousness due to acute global impairment of cerebral blood flow. The onset is rapid, duration is brief, and recovery is often spontaneous and complete. Cough syncope, which occurs immediately after coughing, is a well-recognized condition that has remained incompletely understood despite a number of studies examining its pathophysiology [10-16]. Several mechanisms have been suggested in this regard. With continuous coughing, intra-thoracic pressure increases resulting in decreased venous return and decreased cardiac output [11]. Since cough syncope may occur without prolonged period of coughing, it has been suggested that baroreceptor mechanism via a baroreceptor-initiated neural vasodilation-bradycardia reflex, could be partly responsible for the syncope. Hence, subjects susceptible for cough syncope exhibit greater hypotensive response to transient exaggerated intra-arterial pressure pulses. Both the magnitude and duration of the consequent hypotension are greater than in other fainters, and the hypotensive-triggered compensatory positive chronotropic response is absent or markedly suppressed $[10,11]$. Other suggested mechanism indicates that the increased intra-thoracic pressure during coughing is transmitted to the cerebrospinal fluid, causing an acute pressure increase in the skull, compromising cerebral perfusion and resulting in syncope $[12,17]$. Synergistic effect between jugular venous reflex and plasma endothelin-1 levels [18], and carotid sinus hypersensitivity [16], complete atrioventricular block [15] and sinus arrest [19], has also been suggested.

Despite widespread vaccination, Bordetella pertussis is increasingly identified as a cause of subacute and chronic cough in adolescents and adults over the last 20 years [20]. In the pre-vaccine era, more than $90 \%$ of reported cases of pertussis occurred in children younger than 10 years old. Currently, about half of the reported cases occur in adolescents and adults [21]. The clinical characteristics of pertussis among adults are less obvious than among children. While the clinical presentation in children classically consists of three phases (catarrhal, paroxysmal and convalescent) [21], in adults it is usually a long and mild disease with prolonged cough, sometimes a whooping cough, rhinorrhea, low grade temperature, sleep disturbances and cough syncope [22].

Until yet, only five cases of cough syncope related to pertussis have been described in the medical literature [6-9], two of which are not well documented [9]. The pathogenic mecha- nism responsible for cough syncope in these cases has not been mentioned or evaluated. In our patient, ECG and blood pressure monitoring did not show hypotension or bradycardia during the syncopal attacks. Thus, significant decrease of cerebral perfusion due to acute pressure increase in the skull and of brain venous return could be a possible pathogenic mechanism of the cough syncope.

Pertussis is increasingly reported as cause of subacute and chronic cough in adolescents and adults with a rate of about $50 \%$ of the reported cases. Since cough syncope is not rare, and the clinical characteristics of pertussis among adults are less obvious than among children, where in adults the disease is usually long and mild with prolonged cough, physicians should be alert to the possibility that in adults with prolonged cough with or without syncope, the cough attacks could be related to presence of pertussis. By proper antibiotic treatment of their pertussis, the syncopal attacks induced by their cough could be prevented as happened with our patient.

\section{Author Contributions}

All authors contributed significantly to the collection of data and preparing of the manuscript.

\section{Financial Support}

None.

\section{Conflict of Interest}

The authors declare no conflict of interest.

\section{References}

1. Krediet CT, Wieling W. Edward P. Sharpey-Schafer was right: evidence for systemic vasodilatation as a mechanism of hypotension in cough syncope. Europace. 2008;10(4):486-488.

2. Kerr A, Jr., Derbes VJ. The syndrome of cough syncope. Ann Intern Med. 1953;39(6):1240-1253.

3. Kusuyama T, Iida H, Kino N, Shimodozono S, Kanazawa Y. Cough syncope induced by gastroesophageal reflux disease. J Cardiol. 2009;54(2):300-303.

4. Maznyczka A, Squire IB. Cough syncope--a diagnosis under pressure. Lancet. 2010;376(9739):486.

5. Zeng W, Deng H. Cough syncope: constrictive pericarditis. Intern Med. 2013;52(4):463-465.

6. Jenkins P, Clarke SW. Cough syncope: a complication of adult whooping cough. Br J Dis Chest. 1981;75(3):311313.

7. Yoshida S, Nakayama T. [Cough syncope syndrome caused by pertussis]. Nihon Naika Gakkai Zasshi. 2003;92(7):1321-1324.

8. Pertussis outbreak among adults at an oil refinery--Illi- 
nois, August-October 2002. MMWR Morb Mortal Wkly Rep. 2003;52(1):1-4.

9. Jain AM. Cough syncope. Indian J Pediatr. 1971;38(286):434-436.

10. Benditt DG, Samniah N, Pham S, Sakaguchi S, Lu F, Lurie $\mathrm{KG}$, Ermis $\mathrm{C}$. Effect of cough on heart rate and blood pressure in patients with "cough syncope". Heart Rhythm. 2005;2(8):807-813.

11. Sharpey-Schafer EP. The mechanism of syncope after coughing. Br Med J. 1953;2(4841):860-863.

12. McIntosh HD, Estes EH, Warren JV. The mechanism of cough syncope. Am Heart J. 1956;52(1):70-82.

13. Keer A, Jr., Eich RH. Cerebral concussion as a cause of cough syncope. Arch Intern Med. 1961;108:248-252.

14. Pedersen A, Sandoe E, Hvidberg E, Schwartz M. Studies on the mechanism of tussive syncope. Acta Med Scand. 1966;179(6):653-661.

15. Hart G, Oldershaw PJ, Cull RE, Humphrey P, Ward D. Syncope caused by cough-induced complete atrioventricular block. Pacing Clin Electrophysiol. 1982;5(4):564566.

16. Wenger TL, Dohrmann ML, Strauss HC, Conley MJ,
Wechsler AS, Wagner GS. Hypersensitive carotid sinus syndrome manifested as cough syncope. Pacing Clin Electrophysiol. 1980;3(3):332-339.

17. Mattle HP, Nirkko AC, Baumgartner RW, Sturzenegger M. Transient cerebral circulatory arrest coincides with fainting in cough syncope. Neurology. 1995;45(3 Pt 1):498-501.

18. Chung CP, Cheng CY, Zivadinov R, Chen WC, Sheng $\mathrm{WY}$, Lee $\mathrm{YC}, \mathrm{Hu} \mathrm{HH}$, et al. Jugular venous reflux and plasma endothelin-1 are associated with cough syncope: a case control pilot study. BMC Neurol. 2013;13:9.

19. Aliyev F, Kilickesmez KO, Celiker C, Turkoglu C. Cough-induced sinus arrest resulting in recurrent episodes of syncope: is it really transient? J Cardiovasc Med (Hagerstown). 2012;13(7):468-470.

20. Gregory DS. Pertussis: a disease affecting all ages. Am Fam Physician. 2006;74(3):420-426.

21. Cornia PB, Hersh AL, Lipsky BA, Newman TB, Gonzales R. Does this coughing adolescent or adult patient have pertussis? JAMA. 2010;304(8):890-896.

22. Boulouffe C, Vanpee D. Increasing cause of cough among adults. Emerg Med Australas. 2008;20(3):280-283. 